\title{
Functional outcomes of multi-condition collaborative care and successful ageing: results of randomised trial
}

\author{
(?) $(\Theta \Theta$ OPEN ACCESS
}

\author{
Michael Von Korff senior investigator ${ }^{1}$, Wayne J Katon professor ${ }^{2}$, Elizabeth H B Lin affiliate \\ investigator $^{1}$, Paul Ciechanowski associate professor ${ }^{2}$, Do Peterson biostatistician ${ }^{1}$, Evette J Ludman \\ research associate ${ }^{1}$, Bessie Young associate professor ${ }^{3}$, Carolyn M Rutter senior investigator ${ }^{14}$
}

${ }^{1}$ Group Health Research Institute, 1730 Minor Avenue, Seattle, WA 98101, USA; ${ }^{2}$ Department of Psychiatry and Behavioral Sciences, University of Washington School of Medicine, Seattle, Washington; ${ }^{3}$ Veteran's Affairs Puget Sound Health Care System and Division of Nephrology, Department of Medicine, University of Washington School of Medicine, Seattle; ${ }^{4}$ Department of Biostatistics, University of Washington School of Public Health, Seattle

\begin{abstract}
Objective To evaluate the effectiveness of integrated care for chronic physical diseases and depression in reducing disability and improving quality of life.

Design A randomised controlled trial of multi-condition collaborative care for depression and poorly controlled diabetes and/or risk factors for coronary heart disease compared with usual care among middle aged and elderly people
\end{abstract}

Setting Fourteen primary care clinics in Seattle, Washington.

Participants Patients with diabetes or coronary heart disease, or both, and blood pressure above $140 / 90 \mathrm{~mm} \mathrm{Hg}$, low density lipoprotein concentration $>3.37 \mathrm{mmol} / \mathrm{L}$, or glycated haemoglobin $8.5 \%$ or higher, and PHQ-9 depression scores of $\geq 10$.

Intervention A 12 month intervention to improve depression, glycaemic control, blood pressure, and lipid control by integrating a "treat to target" programme for diabetes and risk factors for coronary heart disease with collaborative care for depression. The intervention combined self management support, monitoring of disease control, and pharmacotherapy to control depression, hyperglycaemia, hypertension, and hyperlipidaemia.

Main outcome measures Social role disability (Sheehan disability scale), global quality of life rating, and World Health Organization disability assessment schedule (WHODAS-2) scales to measure disabilities in activities of daily living (mobility, self care, household maintenance).

Results Of 214 patients enrolled (106 intervention and 108 usual care), disability and quality of life measures were obtained for 97 intervention patients at six months (92\%) and 92 at 12 months (87\%), and for 96 usual care patients at six months (89\%) and 92 at 12 months (85\%). Improvements from baseline on the Sheehan disability scale (-0.9, 95\% confidence interval -1.5 to $-0.2 ; P=0.006$ ) and global quality of life rating $(0.7,0.2$ to $1.2 ; P=0.005)$ were significantly greater at six and 12 months in patients in the intervention group. There was a trend toward greater improvement in disabilities in activities of daily living $(-1.5,-3.3$ to 0.4 ; $P=0.10)$.

Conclusions Integrated care that covers chronic physical disease and comorbid depression can reduce social role disability and enhance global quality of life.

Trial registration Clinical Trials NCT00468676.

\section{Introduction}

Depression is more common among people with chronic physical conditions. ${ }^{1}$ When both mental and physical disorders are present, the risks of disability in carrying out activities in family and household life are increased. ${ }^{2-4}$ As people live longer in both developed and developing countries, the prevalence of chronic physical disease accompanied by depression is likely to grow in proportion. ${ }^{5}$ For these reasons, improving health outcomes of people with chronic physical disease, particularly those with psychological illness as well, is a major challenge for improving the health of ageing populations worldwide..$^{6-9}$ Delay of the harmful effects of growing older has been called "successful aging," "healthy aging," and "compression of morbidity." ${ }^{10-13}$ Health promotion and disease prevention interventions are often cited as effective strategies to enhance successful ageing. For example, healthy diet, vigorous physical activity, sustained mental activity, and effective medical care are believed to promote successful ageing. ${ }^{10-14}$ The potential of effective medical care to promote successful ageing among people with major chronic conditions, however, has received less attention than health promotion and disease prevention strategies.

Although it is well established that depression impedes successful ageing, ${ }^{15}{ }^{16}$ the potential for treatment of depression 
to enhance ageing among people with chronic physical disease is often overlooked. Such treatment has been found to reduce disability among patients with arthritis, ${ }^{17}$ diabetes,,${ }^{18} 19$ heart disease, ${ }^{20}{ }^{21}$ and chronic pain. ${ }^{22}$ Integration of treatment for depression into the care of chronic physical disease could provide a means to enhance ageing among chronically ill people. ${ }^{23}$ Improved control of risk factors for coronary heart disease, including blood pressure, glycaemic control among people with diabetes, and low density lipoprotein cholesterol concentration, enhances successful ageing by preventing major cardiovascular and cerebrovascular events. ${ }^{24}$ The associated benefits of improved control of risk factors for coronary heart disease on functional status and quality of life, however, are less clear. While antihypertensive drugs can have negative effects on specific dimensions of quality of life (such as sexual function), ${ }^{25}$ large trials of management of hypertension have found either no overall effects on quality of life or modest gains. ${ }^{26}$ The ACCORD trial of intensive glycaemic control among people with diabetes, which was stopped early because of increased mortality in the intensive management group, did not find clinically meaningful effects of intensive control on health related quality of life. ${ }^{27}$ Thus, previous research suggests that treatment for depression might have more robust short term effects in improving functional outcomes among patients with chronic disease, whereas efforts to improve control of risk factors for coronary heart disease can have long term benefits through preventing major cardiovascular and cerebrovascular events.

We evaluated the effects on function and health related quality of life of an integrated care management intervention for chronic physical disease and depression. ${ }^{28}$ The intervention managed inadequate blood pressure, glycaemic, and lipid control among patients with diabetes or heart disease, or both, along with managing comorbid major depression. In contrast with previous evaluations of depression treatment among patients with chronic disease, integrated care simultaneously covered physical and psychological aspects of illness in combination. We assessed whether integrated care was associated with reduced disability and improved quality of life, thereby contributing to successful ageing among people with poorly controlled chronic physical disease. In initial reports from this trial, we found that integrated care reduced depression and improved self monitoring and management of drugs, ${ }^{29}$ resulting in improved glycaemic, blood pressure, and lipid control, ${ }^{30}$ thereby affording a more favourable profile for long term risks for major cardiovascular and cerebrovascular events. We have now examined effects of integrated care on disability and quality of life outcomes among depressed patients with poorly controlled diabetes or risk factors for coronary heart disease, or both.

\section{Methods}

Participants were recruited from May 2007 to October 2009 in 14 Group Health primary care clinics.

\section{Study participants}

Study participants included adult men and women with a mean age of 57.4 (SD 10.5) in the intervention group and 56.3 (SD 12.1) in the usual care group. We identified patients with diabetes or coronary heart disease, or both, and blood pressure above 140/90 mm $\mathrm{Hg}$, low density lipoproteins above 3.37 $\mathrm{mmol} / \mathrm{L}$, or glycated haemoglobin $\left(\mathrm{HbA}_{1 \mathrm{c}}\right)$ of $8.5 \%$ or higher. Eligible patients were ambulatory, spoke English, and planned to continue Group Health enrolment for 12 months. Exclusion criteria were terminal illness, residence in long term care, severe hearing loss, planned bariatric surgery, pregnant or nursing, ongoing psychiatric care, bipolar disorder or schizophrenia, use of antipsychotic drugs or mood stabilisers, and mental confusion suggesting dementia. Eligible patients received the patient health questionnaire- $2^{31}$ depression screen by post or telephone and, if the results were positive, the patient health questionnaire-9 by telephone interview at a subsequent assessment. A score of 3 or higher on the patient health questionnaire- 2 and 10 or higher on the patient health questionnaire- 9 was required for eligibility. Eligible patients completed a baseline interview and gave verbal consent for laboratory tests before an in-person visit, at which they provided written informed consent before randomisation.

\section{Randomisation and treatment assignment}

Patients were assigned to treatment group with a permuted block design with randomly selected block sizes of four, six, and eight patients. After baseline evaluation, a study nurse contacted patients assigned to the intervention to initiate treatment. After randomisation, patients in the usual care group were advised to consult with their primary care physician to receive care for depression, diabetes, or coronary heart disease. With permission from the patient, primary care physicians of those in the usual care group were notified about depression and measures of poor disease control. Primary care physicians also received results of the six and 12 month laboratory tests.

\section{Intervention}

A 12 month intervention, called TEAMcare, sought to improve depression and glycaemic, blood pressure, and lipid control by integrating a treat to target ${ }^{32}$ programme for diabetes and coronary heart disease with collaborative care for depression. ${ }^{28} 29$ The intervention combined self management support, monitoring of indicators of disease control, and drugs to control depression, hyperglycaemia, hypertension, and hyperlipidaemia. Patients worked collaboratively with intervention nurses and primary care physicians to create individualised clinical and self management goals. In structured visits in the patient's primary care clinic every one to three weeks, nurses monitored patients' progress in depression (patient health questionnaire-9), control of medical diseases, and self care activities. Treatment protocols, with commonly used drugs, guided adjustments in treatment for patients not achieving specific goals.

Nurses followed patients proactively to support adherence to drugs, using motivational coaching methods. ${ }^{29}{ }^{33}$ Patients received self care materials including: The Depression Helpbook, ${ }^{34}$ a depression care DVD, a chronic disease management booklet and other materials, and self monitoring devices (blood pressure or blood glucose meters) appropriate to their condition. Nurses received weekly supervision with a psychiatrist (WK or PC), a primary care physician (EHBL or $\mathrm{BY}$ ), and a psychologist (EJL) to review new cases and progress of patients. An electronic registry supported tracking patient health questionnaire-9 scores and concentrations of glycated haemoglobin and low density lipoprotein and blood pressure. The multidisciplinary consultants recommended initial choices and changes in drugs tailored to the patient's history and clinical response. The nurse communicated recommendations in changes in treatment to the primary care physician responsible for managing drug treatment. Once patients achieved targeted levels for relevant measures, the nurse and patient developed a maintenance plan. The nurses then followed up patients with telephone calls every four to six weeks to complete a patient health questionnaire- 9 and review adherence and laboratory test results. Patients whose disease control worsened were offered 
follow-up and protocol based regimens for intensification of treatment.

\section{Disability and quality of life}

Disability in carrying out life activities was assessed with the Sheehan disability scale. ${ }^{35}$ Participants rated the extent to which health interfered with work, family life, and social life on scales of 0 to 10 , where 0 was not at all and 10 was unable to carry on any activities. The World Health Organization disability assessment schedule (WHODAS-2) ${ }^{36}$ was used to assess disabilities in activities of daily living, including subscales for mobility, self care, and household maintenance. Each item was rated none (0), mild (1), moderate (2), severe (3), or extreme/can't do (4). The schedule was scored by summing the 15 items (range 0-60). We also administered an item assessing days of reduced household work because of health. This item has been used to monitor disability levels in populations, but it is highly skewed limiting sensitivity to change. ${ }^{37}$ Participants rated global quality of life, including physical and mental wellbeing, on a 0 to 10 scale, where 0 was "bad as you can imagine" and 10 was "perfect." A single item quality of life rating has been found to correlate highly with longer scales but might be less precise than multi-item scales. ${ }^{38}$

\section{Disease control}

At baseline and six and 12 months, telephone interviewers assessed symptoms of depression, disability, quality of life, and other study measures. Depression was assessed by the SCL-20. ${ }^{39}$ Blood pressure and glycated haemoglobin were measured in person at baseline and six and 12 months and fasting low density lipoprotein concentration at baseline and 12 months.

\section{Study oversight}

A data safety monitoring board initially reviewed methods and reviewed outcomes every six months thereafter.

\section{Statistical analyses}

We describe disability and quality of life outcomes at six and 12 months after randomisation using linear regression models adjusted for baseline values. Models that combine information across time points were estimated with general estimating equations to account for correlation. All analyses were based on the treatment group to which the participant was randomly assigned (intent to treat).

\section{Results}

Of 214 patients enrolled (106 intervention and 108 usual care), $88 \%$ completed all six month telephone and laboratory assessments and $83 \%$ completed all 12 month assessments. Patients in both groups were similar at baseline. ${ }^{24} \mathrm{We}$ obtained measures on disability and quality of life for 97 patients at six months (92\%) and 92 at 12 months (87\%) in the intervention group and 96 at six months (89\%) and 92 at 12 months (85\%) in the usual care group. The CONSORT diagram for this trial is available elsewhere. ${ }^{30}$

As previously reported, patients in the intervention group showed significantly greater overall improvement than controls across glycated haemoglobin concentration, low density lipoprotein concentration, systolic blood pressure, and SCL-20 depression outcomes. ${ }^{30}$ The unadjusted differences in change from baseline to 12 month follow-up between the intervention and control groups were $0.58 \%$ for glycated haemoglobin, 0.18 $\mathrm{mmol} / \mathrm{L}$ for low density lipoprotein, $5.1 \mathrm{~mm} \mathrm{Hg}$ for systolic blood pressure, and 0.40 for SCL-20 depression score (effect size 0.67 ). These differences are comparable with effects observed in collaborative care trials targeting single conditions. Improvement from baseline on the Sheehan disability scale $(\mathrm{P}=0.006)$ and global quality of life rating $(\mathrm{P}=0.005)$ were significantly greater at six and 12 months in patients in the intervention group than among patients in the usual care group (table $\downarrow$ ). After adjustment for baseline differences, at 12 months the effect size was 0.30 for the Sheehan disability scale and 0.39 for intervention effect on the global quality of life rating. The WHODAS-2 measure of disabilities in activities of daily living showed a modest non-significant trend towards greater improvement in the intervention group than in the usual care control group $(\mathrm{P}=0.10)$. The effect size for intervention effect for disabilities in activities of daily living was 0.12 at 12 months. There was no difference in restricted days of household maintenance activities between the two groups: mean 8.9 $(\mathrm{SD}=10.2)$ at baseline, $6.4(\mathrm{SD}=8.7)$ at six months, and 6.4 $(\mathrm{SD}=9.2)$ at 12 months in the intervention group and 8.4 $(\mathrm{SD}=10.0), 5.6(\mathrm{SD}=8.7)$, and 6.7 $(\mathrm{SD}=9.3)$, respectively, in the usual care group. The estimated mean difference was 0.0 for six and 12 months $(-0.3$ to $0.4 ; \mathrm{P}=0.8)$.

We examined confidence intervals of the estimated mean differences (figure $\Downarrow$ ), which were of moderate size for the Sheehan disability score and global quality of life rating. The confidence intervals of the estimated mean difference for the WHODAS-2 measure of disabilities in activities of daily living were larger.

\section{Discussion}

\section{Principal findings}

Among people with poorly controlled chronic physical disease, ageing can be accompanied by loss of function, reduced self efficacy, and increased risk of depressive illness. An integrated intervention for depression and chronic disease control can reduce social role disability and improve global quality of life among patients with depression and poorly controlled diabetes or coronary heart disease, or both. Disabilities in activities of daily living (mobility, self care, household maintenance) showed a possible trend towards improved functioning, but the effect size was small. Restricted activity days for household maintenance did not show an intervention effect. Previous trials of disease management interventions to improve control of hypertension or glycaemic control have not found consistent substantial associated benefits for functional status or health related quality of life..$^{25-27}$

\section{Strengths and weaknesses}

Evaluation of confidence intervals for the mean differences between intervention and control groups showed moderate precision for evaluation of social role disability and global quality of life outcomes. The uncertain and small benefits for disabilities in activities of daily living indicate that approaches to strengthening intervention effects for such disabilities, such as targeting of specific tasks among patients with considerable disabilities, might be needed.

As this trial had a smaller sample size than recommended for evaluation of disability outcomes, ${ }^{40}$ cautious interpretation is warranted. Potential benefits of integrated care for physical and psychological comorbidities need to be evaluated across multiple trials. Results for benefits on functional disability, however, have been consistently observed across previous trials of treatment for depression in patients with chronic disease. ${ }^{17-22}$ 
These consistent results suggest that treating depression as part of a multi-condition collaborative care intervention could yield improved quality of life and reduced social role disability, as well as enhanced disease control and reduced depression.

The TEAMcare intervention included elements that sought to empower participants, including motivational enhancement, activation, problem solving, and scheduling of pleasant events, as well as improved drug treatment for depression. Depression treatment might directly improve functional outcomes by increasing energy, self confidence, and interest in life activities. We could not determine which components of a multifaceted intervention were effective in improving functional outcomes.

\section{Implications}

Given ageing populations with an increasing prevalence of chronic disease, successful ageing in people with poorly controlled chronic physical disease and comorbid depression is of growing importance. ${ }^{78}$ As approaches to enhancing successful ageing have tended to emphasise health promotion and strategies for prevention of disease, the associated benefits of depression treatment for successful ageing among people with comorbid chronic physical and psychological illnesses might have received less attention than warranted. Control of risk factors for coronary heart disease to prevent major cardiovascular and cerebrovascular events is consistent with the traditional emphasis on preventive and health promotion strategies for enhancing successful ageing. Integrated care for chronic disease and comorbid psychological illness, however, might have associated benefits for successful ageing by improving health related quality of life and functional status in the short term. We conclude that successful ageing could be enhanced by integrated care of multiple chronic conditions that covers both physical and psychological illness.

In a cost effectiveness evaluation of the TEAMcare intervention it was found that intervention patients had a mean of 114 more days without depression over a two year period, and a projected 0.335 additional quality adjusted life years (QALYs) based on the predicted effect of improved disease control on QALYs. ${ }^{41}$ The total outpatient costs of intervention patients (including intervention costs) over a two year period averaged at \$594 $(£ 377, € 430)$ less than the outpatient healthcare costs of control patients, but the confidence intervals for the cost per depression-free day were wide (mean $\$ 5.94,95 \%$ confidence interval $-\$ 29.96$ to $\$ 19.17$ ). The $95 \%$ confidence interval for the cost per QALY ranged from $-\$ 2878$ to $\$ 2878$. This cost effectiveness analysis did not consider the effects of the intervention on current measures of functional status or quality of life.

Depression is associated with social isolation, decreased energy and motivation, poor self esteem, and interpersonal difficulties. By covering these psychosocial impairments, depression treatment can reduce disability and improve quality of life among people whose depression occurs within the context of a chronic physical disease.

\section{Future research}

Large scale evaluation of practical approaches to implementing integrated care for multiple conditions is needed. Large scale trials should assess benefits for social role disability and quality of life outcomes, as well as improved control of chronic physical and psychological conditions. If our results can be replicated and extended on a larger scale, the TEAMcare intervention could provide a means of enhancing successful ageing among people with poorly controlled chronic physical disease and comorbid psychological illness.

The TEAMcare study would not have been possible without the support and participation of Group Health patients, primary care physicians, consultants, and leaders. We also acknowledge efforts and expertise by Tara Beatty, Malia Oliver, Sue Ruedebusch, Diana Griffith, and Sandy Randles. We also thank Michelle Wong, R James Dudl, and Kaiser Permanente Care Management Institute for providing the treat to target diabetes guidelines that we adapted. The content is solely the responsibility of the authors and does not necessarily represent the official views of the organisations that employ them.

Contributors: MVK drafted the paper, planned and interpreted analyses, assisted in securing funding for the trial, and shared responsibility for direction of the trial. WJK is the guarantor of the study, obtained funding for the trial, directed execution of the trial, participated in the design and provision of the intervention, assisted in planning analyses for this paper, and reviewed and edited drafts of this paper. EHBL co-led work on designing and implementing the intervention, and reviewed and edited drafts of this paper. PC, EJL, and BY participated in the design and implementation of the intervention and reviewed and edited drafts of this paper. PC participated in the design and implementation of the intervention and reviewed and edited drafts of this paper. CMR and DP were responsible for planning, implementing, and interpreting statistical analyses for this paper, and reviewed and edited drafts of this paper.

Funding: This study was supported by grants MH041739 and MH069741 from the National Institute of Mental Health Services Division, Bethesda, MD (WJK) and by institutional support from Group Health Cooperative. The sponsors of this research had no influence over the conduct of the study, the analyses of study data, or the content of this paper.

Competing interests: All authors have completed the ICMJE uniform disclosure form at www.icmje.org/coi_disclosure.pdf (available on request from the corresponding author) and declare: no support from any organisation for the submitted work; no financial relationships with any organisations that might have an interest in the submitted work in the previous three years; WJK serves on boards for Eli Lilly and Wyeth and has given lectures for Eli Lilly, Wyeth, Forest, and Pfizer; EHBL serves on the board of the physician postgraduate press external advisory board, has given lectures for the University of Washington relevant to dissemination of the IMPACT and TEAMcare interventions, and developed a CME on adherence for Health Star Communications; $\mathrm{PC}$ is the founder of Samepage, an organisation that provides consultation and educational tools for improving patient-provider relationships.

Ethical approval: The trial was approved by the Group Health Institutional Review Board, and informed consent was given by all patients before enrolment in the trial.

Data sharing: Requests for analyses of de-identified data from this trial should be directed to Wayne Katon (wkaton@u.washington.edu).

1 Gureje $\mathrm{O}$. The pattern and nature of mental-physical comorbidity: specific or general. In: Von Korff M, Scott KM, Gureje O, eds. Global perspectives on mental-physical comorbidity in the WHO world mental health surveys. Cambridge University Press, 2009:51-83.

2 Scott KM, Von Korff M, Alonso J, Angermeyer MC, Bromet E, Fayyad J, et al. Mental-physical co-morbidity and its relationship with disability: results from the World Mental Health Surveys. Psychol Med 2008;26:1-11.

3 Von Korff M, Ormel J, Katon W, Lin EHB. Disability and depression in medical patients: a longitudinal analysis. Ach Gen Psychiatry 1992;49:91-100.

4 Ormel J, Von Korff M, Ustun TB, Pini S, Korten A, Oldehinkel T. Common mental disorders and disability across cultures: results from the WHO Collaborative Study on psychological problems in general health care. JAMA 1994;272:1741-8.

5 Von Korff M, Scott KM, Gureje O, eds. Global perspectives on mental-physical comorbidity in the WHO world mental health surveys. Cambridge University Press, 2009.

6 McMichael AJ, McKee M, Shkolnikov V, Valkonen T. Mortality trends and setbacks: global convergence or divergence. Lancet 2004;363:1155-9.

7 Christensen KM, Doblhammer G, Rau R, Vaupel JW. Aging populations: the challenges ahead. Lancet 2009;374:1196-208.

8 Jozan P. The epidemiological future. Health Policy 1991;19:19-32.

8 Jozan P. The epidemiological future. Health Policy 1991;19:19-32. KM, Gureje O, eds. Global perspectives on mental-physical comorbidity in the WHO world mental health surveys. Cambridge University Press, 2009:1-11. 


\section{What is already known on this topic}

Previous trials of disease management interventions to improve blood pressure and glycaemic control have not found consistent associated benefits for functional status or health related quality of life

Treatment for depression improves these outcomes among depressed patients with comorbid chronic physical disease

\section{What this study adds}

Multi-condition collaborative care for depression and poorly controlled diabetes or risk factors for heart disease, or both, resulted in improvements in functional outcomes and health related quality of life as well as improved control of blood pressure, blood glucose lipids, and depressive symptoms

Integrated care for depression and poorly controlled chronic physical disease might enhance successful ageing among people with chronic physical and psychological conditions

10 Rowe JW, Kahn RL. Human aging: usual and successful. Science 1987;237:143-9.

11 Rowe JW, Kahn RL. Successful aging. Gerontologist 1997;37:433-40.

12 Guralnik JM, Kaplan GA. Predictors of healthy aging: prospective evidence from the Alameda County Study. Am J Public Health 1989;79:703-8.

13 Fries JF. Aging, natural death, and the compression of morbidity. N Engl J Med 1980;303:130-5

14 Larson EB. Prospects for delaying the rising tide of worldwide, late-life dementias. Int Psychogeriatr 2010;22:1196-202.

15 Mor V. The compression of morbidity hypothesis: a review of research and prospects for the future. J Am Geriatr Soc 2005;53(9 suppl):S308-9.

16 Depp CA, Jeste DV. Definitions and predictors of successful aging: a comprehensive review of larger quantitative studies. Am J Geriatr Psychiatry 2006;14:6-20.

7 Lin EHB, Katon W, Von Korff M, Tang L, Williams JW, Kroenke K, et al. for the IMPACT investigators. Effect of improving depression care on pain and function among older adults with arthritis: a randomized controlled trial. JAMA 2003;290:2428-34.

18 Williams JW Jr, Katon W, Lin EH, Nöel PH, Worchel J, Cornell J, et al. The effectiveness of depression care management on diabetes-related outcomes in older patients. Ann Intern Med 2004:140:1015-24.

19 Ell K, Katon W, Xie B, Lee PJ, Kapetanovic S, Guterman J, et al. Collaborative care management of major depression among low-income, predominately Hispanic subjects with diabetes. Diabetes Care 2010;33:706-13.

20 Lespérance F, Frasure-Smith N, Koszycki D, Laliberté MA, van Zyl LT, Baker B, et al Effects of citalopram and interpersonal psychotherapy on depression in patients with coronary artery disease. The Canadian Cardiac Randomized Evaluation of Antidepressant and Psychotherapy Efficacy (CREATE) trial. JAMA 2007;297:367-79.

21 Rollman BL, Belnap BH, LeMenager MS, Mazumdar S, Houck PR, Counihan PJ, et al. Telephone-delivered collaborative care for treating post-CABG depression: a randomized controlled trial. JAMA 2009;302:2095-103.

22 Kroenke K, Bair MJ, Damush TM, Wu J, Hoke S, Sutherland J, Tu W. Optimized antidepressant therapy and pain self-management in primary care patients with depression and musculoskeletal pain: a randomized controlled trial. JAMA 2009;301:2099-110.

23 Simon GE. Clinical implications. In: Von Korff M, Scott KM, Gureje O, eds. Global perspectives on mental-physical comorbidity in the WHO world mental health surveys. Cambridge University Press, 2009:297-301.

24 Lewis SJ. An urgent matter-identifying your patients' cardiovascular risk and improving their outcomes. Low-density lipoprotein cholesterol and coronary heart disease: the importance of reaching target goals with statin therapy. J Fam Practice 2009;58:S32-40.

25 Cote I, Gregoire JP, Moisan J. Health-related quality-of-life measurement in hypertension: a review of randomized controlled drug trials. Pharmacoeconomics 2000;18:435-50.

26 Fogari R, Zoppi A. Effect of antihypertensive agents on quality of life in the elderly. Drugs Aging 2004:21:377-93.

27 Anderson RT, Venkat Narayan KM, Feeney P, Goff D, Ali MK, Simmons DL, et al. for the ACCORD Investigators. Effect of intensive glycemic lowering of health-related quality of life in type 2 diabetes. Diabetes Care 2011;34:807-12.

28 Katon W, Lin EH, Von Korff M, Ciechanowski P, Ludman E, Young B, et al.. Integrating depression and chronic disease care among patients with diabetes and/or coronary heart disease: the design of the TEAMcare study. Contemp Clin Trials 2010;31:312-22.
29 Lin EHB, Von Korff M, Ciechanowski P, Peterson D, Ludman EJ, Rutter C, et al. Treatment adjustment and medication adherence for complex patients with diabetes, heart disease and depression: a randomized controlled trial. Ann Fam Med 2011 (in press).

30 Katon WJ, Lin EHB, Von Korff M, Ciechanowski P, Ludman EJ, Young B, et al Multi-condition collaborative care for chronic illness and depression. N Engl J Med 2010;363:2611-20

31 Arroll B, Goodyear-Smith F, Crengle S, Gunn J, Kerse N, Fishman T, et al. Validation of PHQ-2 and PHQ-9 to screen for major depression in the primary care population. Ann Fam Med 2010;8:348-53.

32 Riddle MC, Rosenstock J, Gerich J. The treat-to-target trial: randomized addition of glargine or human NPH insulin to oral therapy of type 2 diabetic patients. Diabetes Care 2003;26:3080-6

33 Rollnick S, Miller W. What is motivational interviewing? Behav Cogn Psychothe 1995;23:325-34

34 Katon W, Ludman E, Simon G. The depression helpbook. Bull Publishing Company, 2003

35 Leon AC, Olfson M, Portera L, Farber L, Sheehan DV. Assessing psychiatric impairment in primary care with the Sheehan disability scale. Int J Psychiatry Med 1997;27:93-105.

36 Ustun TB, Chatterji S, Kostanjsek N, Rehm J, Kennedy C, Epping-Jordan J, et al. Developing the World Health Organization disability assessment schedule 2.0. Bull World Health Organ 2010;88:815-23.

37 Scholes D, La Croiz AZ, Wagner EH, Grothaus L, Hecht JA. Tracking progress toward national health objective in the elderly: what do restricted activity days signify? Am J Public Health 1991:81:485-8.

38 Lindholt JS, Ventegodt S, Henneberg EW. Development and validation of QoL5 for clinical databases. A short, global and generic questionnaire based on an integrated theory of the quality of life. Eur $J$ Surgery 168:107-13.

39 Derogatis LR, Lipman RS, Rickels K, Uhlenhuth EH, Covi L. The Hopkins symptom checklist (HSCL). A measure of primary symptom dimensions. Mod Probl Pharmacopsychiatry 1974;7:79-110.

40 Sturm $\mathrm{R}$, Unutzer $\mathrm{J}$, Katon $\mathrm{W}$. Effectiveness research and implications for study design: sample size and statistical power. Gen Hosp Psychiatry 1999;21:274-83.

41 Katon W, Russo J, Lin E, Schmittdiel J, Ciechanowski P, Ludman E, et al. Cost-effectiveness of a multicondition collaborative care intervention. Arch Gen Psychiatry 2011 (in press).

Accepted: 16 September 2011

\section{Cite this as: BMJ 2011;343:d6612}

This is an open-access article distributed under the terms of the Creative Commons Attribution Non-commercial License, which permits use, distribution, and reproduction in any medium, provided the original work is properly cited, the use is non commercial and is otherwise in compliance with the license. See: http://creativecommons.org/licenses/by$\mathrm{nc} / 2.0 /$ and http://creativecommons.org/licenses/by-nc/2.0/legalcode. 


\section{Table}

Table 1/ Functional outcome measures according to integrated care (intervention) or usual care at $\mathbf{6}$ and 12 months

\begin{tabular}{|c|c|c|c|c|c|c|}
\hline \multirow[b]{2}{*}{ Outcome } & \multicolumn{2}{|c|}{ Integrated care } & \multicolumn{2}{|c|}{ Usual care } & \multicolumn{2}{|c|}{$P$ value for differences } \\
\hline & No of patients & Mean (SD) & No of patients & Mean (SD) & 12 months & 6 and 12 months \\
\hline \multicolumn{7}{|c|}{ Sheehan social role disability scale $(0-10)$ : higher rating indicates greater disability } \\
\hline Baseline & 106 & $5.6(2.4)$ & 107 & $5.1(2.6)$ & - & - \\
\hline 6 months & 97 & $3.7(3.2)$ & 96 & $4.2(2.6)$ & - & \multirow[t]{2}{*}{0.006} \\
\hline 12 months & 92 & $3.8(3.0)$ & 92 & $4.5(2.9)$ & 0.015 & \\
\hline
\end{tabular}

Global quality of life rating (0-10): higher rating indicates greater quality of life

\begin{tabular}{lllllll}
\hline Baseline & 106 & $4.2(1.9)$ & 107 & $4.7(1.8)$ & - \\
\hline 6 months & 97 & $5.8(2.4)$ & 96 & $5.2(1.8)$ & - & 0.005 \\
\hline 12 months & 92 & $6.0(2.2)$ & 92 & $5.2(1.9)$ & 0.010 & \\
\hline
\end{tabular}

WHODAS-2 activities of daily living (0-4): higher rating indicates greater disability

\begin{tabular}{lcccccc}
\hline Baseline & 105 & $15.8(9.6)$ & 108 & $13.8(9.6)$ & - & - \\
\hline 6 months & 97 & $12.3(10.7)$ & 96 & $12.4(9.8)$ & - & 0.1 \\
\hline 12 months & 92 & $12.9(10.0)$ & 92 & $12.9(11.2)$ & 0.2 & \\
\hline
\end{tabular}

WHODAS=World Health Organization disability assessment schedule. 


\section{Figure}

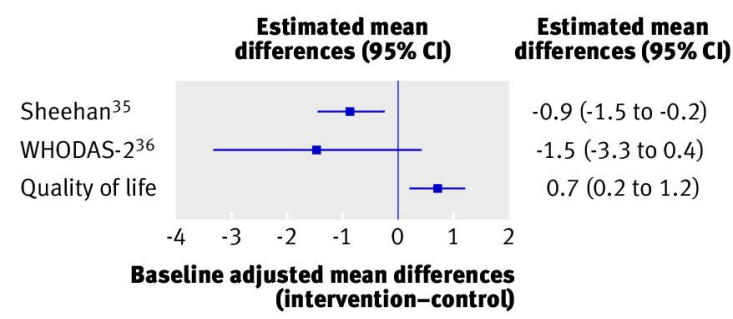

Disability and quality of life outcomes. Mean differences $<0$ on Sheehan disability scale and WHODAS-2 indicate reduced disability levels, while mean difference $>0$ on QOL measure indicates improved quality of life 